\title{
Evaluation of Bean and Soy Tempeh Influence on Intestinal Bacteria and Estimation of Antibacterial Properties of Bean Tempeh
}

\author{
MACIEJ KULIGOWSKI ${ }^{1 *}$ IWONA JASIŃSKA-KULIGOWSKA² and JACEK NOWAK ${ }^{1}$
}

\author{
${ }^{1}$ Department of Fermentation and Biosynthesis, Institute of Food Technology of Plant Origin \\ Faculty of Food Science and Nutrition, Poznań University of Life Sciences, Poznań, Poland \\ ${ }^{2}$ Department of Functional Food and Commodities, Faculty of Human Nutrition and Consumer Sciences, \\ Warsaw University of Life Sciences, Warszawa, Poland
}

Received 15 October 2012, revised 12 February 2013, accepted 9 April 2013

\begin{abstract}
In this study the effect of bean tempeh on the growth of Bacillus subtilis, Escherichia coli, Lactobacillus acidophilus and Lactobacillus paracasei bacteria was investigated. Antibacterial activity was observed only in relation to the bacteria Bacillus subtilis. The effect of tempeh products on human intestinal microflora was also assessed. Bean and soy tempeh were culinarily processed and next digested in conditions simulating the human digestive tract (one of the digestive tracts was equipped with a mechanism simulating absorption). Soy tempeh stimulated most the growth of bacteria of the genus Bifidobacterium, while bean tempeh that of Escherichia coli. Using simulation of absorption for the digestion of fried soy tempeh resulted in a higher rise in the bacteria count of the genus Lactobacillus, while after digestion of fried bean tempeh the highest increase was recorded for Bifidobacterium and E. coli.
\end{abstract}

Key words: tempeh, human intestine microflora, digestion, antibacterial activity

\section{Introduction}

Tempeh is a traditional, fermented product made from legume seeds which originates from Indonesia. Rhizopus oligosporus fungus strains are used most commonly in the fermentation of tempeh (Nout et al., 2005). Interest in this product has been on the rise in various parts of the world due to its high nutritive value and easy preparation for immediate consumption (convenience food). Tempeh's beneficial effects in counteracting diarrhea in monogastric mammals have been described in the literature. So far, this effect after soy tempeh consumption has been observed in humans (Karyadi and Lukito 2000), rabbits (Karyadi and Lukito 1996; Karmini et al., 1997) and pigs (Kiers et al., 2003). These anti-diarrheal features of tempeh may be related to its antibacterial properties. The first reports on the antibacterial properties of extracts from a substrate fermented by the fungus R. oligosporus appeared in 1969 . Different microorganisms, i.e. Streptococcus cremoris (Wang et al., 1969) or Bacillus subtilis (Kobayasi et al., 1992), were indicated as most susceptible to the activity of this type of antibacterial substances. Later studies on the antibacterial activity of soy tempeh extracts showed slight antibacterial activity in relation to B. subtilis and Bacillus stearothermpohilus (Kiers et al., 2002). Peptides which exhibited antibacterial activity in relation to Bacillus cereus were also found in tempeh (Roubos et al., 2008). However, tempeh isolates were not shown to be able to inhibit the growth of Escherichia coli which causes diarrhea in humans and animals (Kiers et al., 2002). In vitro studies found that tempeh isolates inhibited the cell adhesion of an enterotoxic strain of E. coli to intestinal epithelium cells. However, there are no data indicating which of the substances contained in the tempeh affected this process.

The aim of this research was evaluation of antibacterial properties of bean tempeh and comparing impact of bean and soy tempeh on intestine bacteria. No data on the antibacterial properties of bean tempeh have been found. This study analyzed the influence of soy tempeh and bean tempeh on intestinal microflora with the use of an in vitro digestion model. In the studies published to date, the effect of tempeh products on intestinal microflora has not been described. We evaluated the thesis that antibacterial properties and anti-diarrhea

* Corresponding author: M. Kuligowski, Department of Fermentation and Biosynthesis, Institute of Food Technology of Plant Origin, Faculty of Food Science and Nutrition, Poznań University of Life Sciences, Wojska Polskiego 31, 60-637 Poznań, Poland; phone: +48 61848 72 78; fax: +48-61-8487314; e-mail: maciek@up.poznan.pl 
features are connected and if there is a possibility of replacing soy by beans for the production of tempeh without changing their properties against bacteria.

\section{Experimental}

Materials and Methods

Microorganisms. The Rhizopus oligosporus NRRL 2710 fungus strain came from the Northern Regional Research Laboratory, Peoria, Illinois, USA. Bacillus subtilis DSMZ 347, Lactobacillus paracasei subsp. paracasei DSMZ 20213 and Lactobacillus acidophilus DSMZ 20079 bacteria were obtained from Deustche Sammlung von Mikroorganismen und Zellkulturen, Braunschweig, Germany. Escherichia coli 94 (isolated from the human intestine) came from the Institute of Animal Reproduction and Food Analyses, Polish Academy of Sciences, Olsztyn, Poland.

Production of tempeh. The inoculum for soy and bean seed inoculation was a suspension of R. oligosporus spores cultured on a PDA medium for $72 \mathrm{~h}$. The seeds of soy (Glycine max) cv. Noviko and bean (Phaseolus vulgaris) cv. Igołomska, after hulling and boiling (40 min and $20 \mathrm{~min}$, respectively), were inoculated with R. oligosporus spores and fermented at a temperature of $37^{\circ} \mathrm{C}$ for $24 \mathrm{~h}$.

Microbiological media. B. subtilis DSMZ 347 bacteria were cultured in DSM broth (meat extract $0.3 \%$, peptone $0.5 \%$, agar $1.5 \%)$. E. coli 94 was cultured in a medium containing enriched broth at $1.5 \%$, glucose $1.0 \%$ and agar $1.5 \%$. L. paracasei subsp. paracasei DSMZ 20213 and L. acidophilus DSMZ 20079 were cultured in MRS medium (yeast extract $0.4 \%$, meat extract $0.8 \%$, peptone K $1.0 \%$, glucose $2.0 \%$, ammonium hydrogen citrate $0.2 \%$, dipotassium phosphate $0.2 \%$, sodium acetate $0.5 \%$, magnesium sulfate heptahydrate $0.02 \%$, manganese sulfate tetrahydrate $0.005 \mathrm{~g}$, Tween $800.1 \%$ ).

The following selective media to determine changes in the counts of intestinal bacteria were applied: the Garsh medium (peptone K 2.0\%, agar 1.5\%, lactose $1.0 \%$, sodium acetate $0.6 \%$, yeast extract $0.5 \%$, lithium chloride $0.3 \%, \mathrm{Na}_{2} \mathrm{HPO}_{4} \times 12 \mathrm{H}_{2} \mathrm{O} 0.25 \%, \mathrm{~K}_{2} \mathrm{HPO}_{4} 0.2 \%$, L cysteine $0.04 \%, \mathrm{MgSO}_{4} \times 7 \mathrm{H}_{2} \mathrm{O} 0.012 \%$ ) for Bifidobacterium; agar with kanamycin, aesculin and sodium azide (Merck) for bacteria from the genus Enterococcus; McConkey's medium (peptone K 1.7\%, meat peptone $0.3 \%$, bile salts $0.15 \%, \mathrm{NaCl} 0.5 \%$, lactose $1.0 \%$, methyl violet $0.0001 \%$, neutral red $0.003 \%$, agar $1.3 \%$ ) for E. coli; and the MRS medium for bacteria from the genus Lactobacillus.

Bacterial culture conditions. The bacteria B. subtilis were cultured on agar slants at a temperature of $30^{\circ} \mathrm{C}$ for $48 \mathrm{~h}$. Next they were washed off the slants with $10 \mathrm{ml}$ of physiological saline, followed by washing into $90 \mathrm{ml}$ culture broth with no agar addition.

Further culture was run on a shaker in Erlenmeyer flasks at $120 \mathrm{rpm}$ at $30^{\circ} \mathrm{C}$ for $24 \mathrm{~h}$. E. coli and Lactobacillus were cultured on agar slants at $37^{\circ} \mathrm{C}$ for $48 \mathrm{~h}$. Next the bacteria were washed off the slants with $10 \mathrm{ml}$ of physiological saline, followed by washing into $90 \mathrm{ml}$ of the appropriate medium with no agar added. Further culture was run in Erlenmeyer flasks at $37^{\circ} \mathrm{C}$ for $24 \mathrm{~h}$. The number of cells was 8.7, 8.2, 7.7, and $7.3 \log$ c.f.u./ $\mathrm{ml}$ for B. subtilis, E. coli, L. paracasei, and L. acidophilus, respectively.

Preparation of intestinal microflora. Fecal specimens were collected from three healthy individuals ( 2 women and 1 man), aged 20-28 years old, who had not been treated with antibiotics for at least 3 months prior to the specimen collection and had not reported digestive problems. The fecal specimens were placed under anaerobic conditions immediately after excretion. An identical amount (w/w) of phosphate-carbonate buffer at a temperature of $37^{\circ} \mathrm{C}$ was added to each individual's fecal specimen (Barry et al., 1995). Next the entire mass was homogenized, combined at equal volumes and sieved through a sieve with mesh size of $1 \mathrm{~mm}$. These procedures were performed in a nitrogen atmosphere (Aura et al., 2005).

Preparation of extracts from tempeh. An amount of $10 \mathrm{~g}$ of tempeh was homogenized (homogenizer H-500; Pol-EkoAparatura) with $20 \mathrm{ml}$ of water for 3 minutes at $1500 \mathrm{rpm}$. The homogenisate was filtered through a sterile filter with pore size of $0.22 \mu \mathrm{m}$ (Millipore). The $\mathrm{pH}$ of extracts was measured.

Determining antibacterial activity by agar well and paper discs methods. A $1 \mathrm{ml}$ culture of bacterial cells was flooded with a medium specific for each bacterial strain with an addition of agar. The volume of $0.1 \mathrm{ml}$ tempeh extract was poured into a well $0.5 \mathrm{~cm}$ in diameter. For the paper disc analysis, discs saturated with tempeh extract were placed on medium surfaces. Plates inoculated with B. subtilis were incubated at $30^{\circ} \mathrm{C}$, while the other tested bacteria were cultured at $37^{\circ} \mathrm{C}$. After $24 \mathrm{~h}$, the bacterial growth inhibition zone around the well or disc was measured.

Determining antibacterial activity by turbidimetry. The culture was run in test tubes, where $1 \mathrm{ml}$ of bacterial suspension and $0.5 \mathrm{ml}$ of tempeh extract were added to $8.5 \mathrm{ml}$ of the respective medium. Evaluations were taken at $2 / 3$ of the log growth phase of a given indicator strain. Absorbance was measured at a wavelength of $650 \mathrm{~nm}$ on a spectrophotometer (Helios Delta, Thermo Scientific, USA). Dependence curves of absorbance were determined based on the amount of tested bacterial cells.

Digestion under in vitro conditions. The in vitro digestion model consisted of three simulation stages: 
the stomach, small intestine and large intestine. Tempeh after culinary processing (10 min boiling or $5 \mathrm{~min}$ frying in rapeseed oil at $170-180^{\circ} \mathrm{C}$ ) was comminuted, weighed into $6 \mathrm{~g}$ samples, mixed with $60 \mathrm{ml}$ water and placed in $100 \mathrm{ml}$ flat-bottomed flasks.

Using hydrochloric acid, the mixture was brought to $\mathrm{pH} 2$, next a solution of pepsin was added. After $4 \mathrm{~h}$ sodium hydrogencarbonate was added, then intestinalpancreatic extract was added when the $\mathrm{pH}$ reached 6. Next, using sodium hydrogencarbonate, the $\mathrm{pH}$ was raised to 7.4 and intestinal microflora were added. After $2.5 \mathrm{~h}$ the $\mathrm{pH}$ was set at 8.0 and maintained for $18 \mathrm{~h}$. When $\mathrm{pH}$ was established at 8.0 , vessels with the contents being digested were blown with nitrogen (Gumienna et al., 2007).

In vitro digestion model with simulation of active transport. The digestion process using in vitro simulation of active transport was run identically to conventional digestion up to the stage simulating digestion in the small intestine. Fried tempeh was subjected to digestion under such conditions for $24 \mathrm{~h}$. After introducing intestinal-pancreatic extract as well as intestinal microflora and stabilizing $\mathrm{pH}$, the digested contents were transferred to a set containing a membrane with $3.5 \mathrm{kDa}$ pore size (DispoDialyzer, SpectrumLabs). The closed set was placed in a water bath at $\mathrm{pH}$ of 7.4 and temperature of $37^{\circ} \mathrm{C}$. After $2.5 \mathrm{~h}$ the $\mathrm{pH}$ was raised to 8.0 using sodium hydrogencarbonate. The process was stopped after $18 \mathrm{~h}$.

Evaluation of changes in intestinal microflora. Changes in microorganism counts of selected intestinal microflora groups after the stages of digestion were evaluated by plate culture on selective media according to the Koch method (Goderska et al., 2008). The results were expressed as cell count increase, i.e. the difference between the number of bacterial cells after digestion and the number of bacterial cells at the beginning of digestion (increments).

Statistical analysis. Statistical analysis was carried out using Microsoft Excel 2003 and Statistica 8.0 StatSoft software. All experiments were performed with at least three replicates. The least significant difference (LSD) test and Tukey's test were used to verify differences between the samples.

\section{Results and Discussion}

Antibacterial properties. To date, the antibacterial properties of soy tempeh have been mainly described in the literature (Kiers et al., 2002; Roubos et al., 2008). This study focused on determining such properties in bean tempeh. The tested strains used to determine the antibacterial properties of tempeh were bacteria which can colonize the human intestine (E. coli, L. acidophilus and
L. paracasei subsp. paracasei) as well as B. subtilis, which does not colonize the human digestive tract but its particular sensitivity to the antibacterial agent from R. oligosporus post-culture media was reported (Kobayasi et al., 1992; Kiers et al., 2002). When applying the bean tempeh extracts to determine antibacterial activity using the paper disc and well methods, no such activity was observed in relation to the tested bacterial strains. Kiers et al. (2002), using the disc method, recorded antibacterial activity of soy tempeh against Bacillus stearothermophilus and B. subtilis. The tempeh extracts obtained by these researchers did not exhibit antibacterial activity in relation to E. coli, and only one of the R. oligosporus strains used in tempeh fermentation showed activity against B. subtilis. However, the tempeh extracts caused an inhibition of B. stearothermophilus growth.

The lack of antibacterial activity in the bean tempeh extracts, determined using both the well and paper disc methods, could have been caused by a lower amount of substances displaying antibacterial activity against the tested bacteria forming in the bean tempeh in comparison to the soy tempeh (Kiers et al., 2002).

In this study, using turbidimetry, the antibacterial activity of bean tempeh extracts was determined only in relation to B. subtilis (Fig. 1). Kobayasi et al. (1992) obtained similar results: by using the turbidimetric method they showed the antibacterial activity of the R. oligosporus IFO 8631 post-culture medium on a liquid solution of casein hydrolysate against the B. subtilis strains, whereas they did not find such activity against $E$. coli.

The aim of applying tempeh extracts, determined by the turbidimetry method, differing in fermentation time (samples taken every $4 \mathrm{~h}$ ) was to identify the moment of antibacterial substance formation in the tempeh. The presence of an antibacterial agent against the bacteria B. subtilis could be detected in bean tempeh only after the $12^{\text {th }}$ hour of fermentation (Fig. 1), which was the time of visible hyphae of the fungus $R$. oligosporus forming on the seeds. No effect of the tempeh extracts on the growth of Lactobacillus and E. coli was observed. Tempeh extracts did not contain organic acids which could inhibit growth of B. subtilis cells number. The $\mathrm{pH}$ value did not change significantly during the $24 \mathrm{~h}$ of tempeh fermentation. No publication describing the effect of tempeh on bacteria from the genus Lactobacillus was found in the literature.

So far, two substances originating from the R. oligosporus post-culture media and displaying antibacterial activity have been described. One of these was a protein of $5.5 \mathrm{kDa}$, isolated from the R. oligosporus culture on a substrate of casein hydrolysate (Kobayasi et al., 1992), while the other was a peptide weighing less than $3 \mathrm{kDa}$ found in soy tempeh (Roubos et al., 2008). To date it has not been explained if these proteins were synthesized 


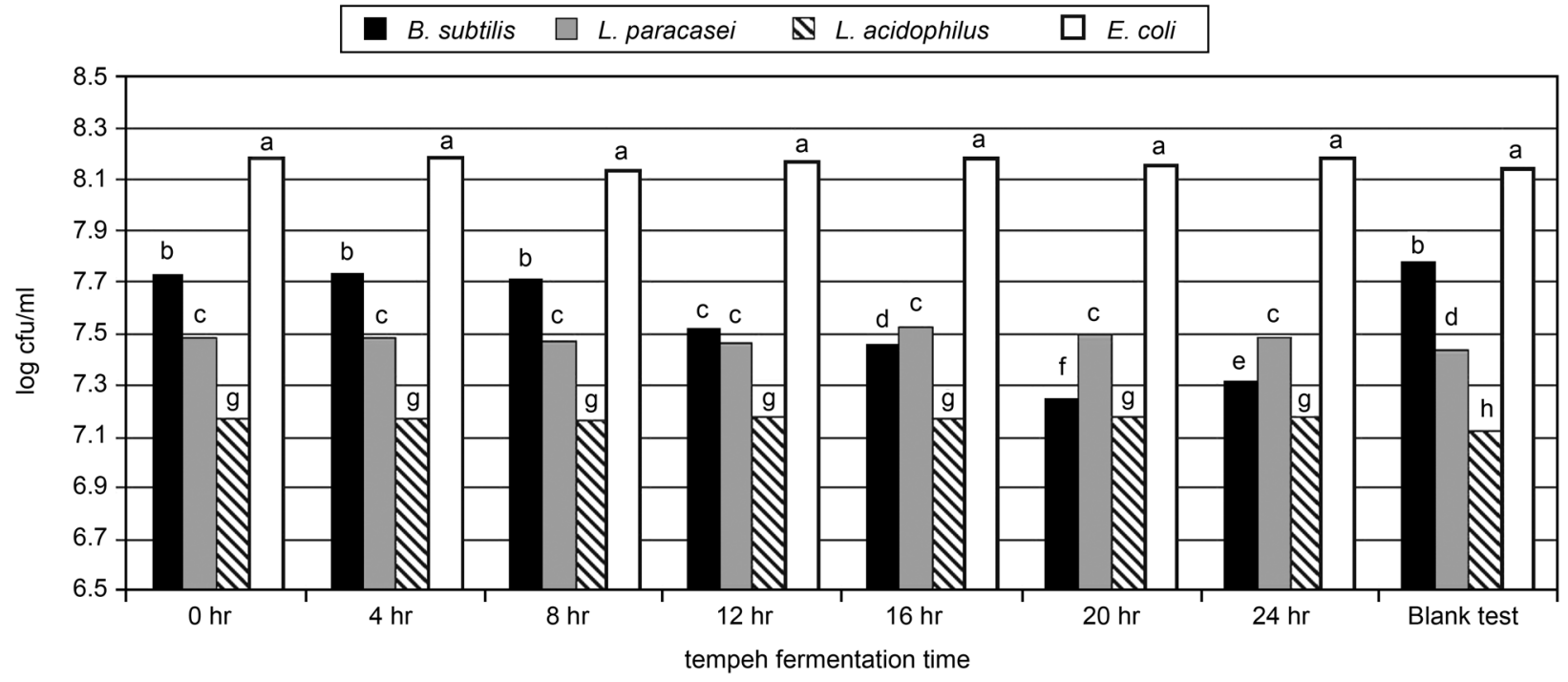

Fig. 1. Antibacterial properties of extracts from bean tempeh fermented for 4 to 24 hours expressed as the decrease of the number $(\log \mathrm{cfu} / \mathrm{ml})$ of test bacteria. Bars marked with different letters differ significantly $(\mathrm{P}<0.05$; LSD test).

by R. oligosporus or whether they could have formed as the result of activity of the enzymatic apparatus of filamentous fungi through the hydrolysis of proteins contained in the fermented substrate.

In the analyzed literature, apart from the antibacterial properties of soy tempeh, antibacterial activity was described only for pea tempeh. Nowak and Steinkraus (1988) evaluated the effect of pea tempeh on the growth and metabolism of Clostridium by using turbidimetry and measuring the amount of gas produced by the tested bacteria. No other publications describing the antibacterial properties of tempeh produced from raw materials other than soy have been found.

Intestinal microflora. Despite reports of tempeh's positive effect in counteracting the onset of diarrhea, to date the influence of tempeh products on intestinal microflora has not been described. We decided to verify whether these properties could be in any way connected with tempeh's influence on the microflora of the human digestive tract.

After $24 \mathrm{~h}$ of fermentation, bean and soy tempeh were subjected to digestion in conditions simulating the human digestive tract. Human fecal microflora were added at the stage corresponding to the small intestine and an increase in the number of microorganisms belonging to the genera Bifidobacterium, Lactobacillus, E. coli and Enterococcus was determined at the end of the digestion process.

Some authors consider McConkey's selective medium, applied as one to proliferate E. coli bacteria, as a medium for the proliferation of bacteria from the group of Enterobacteriaceae (Goderska et al., 2008). However, the manufacturer of this medium (BTL) recommends it as suitable for the proliferation of E. coli, Salmonella and Shigella, which belong to the Enterobac- teriaceae. Due to the considerable dominance of E. coli bacteria over the other microorganisms colonizing the human digestive tract (Marteau et al. 2001) capable of growing on McConkey's medium, bacteria growing on this medium were described as those belonging to E. coli bacteria.

The results of determining the effect of tempehtype products on intestinal microflora are presented as an increment in the count of cells after the completion of digestion in relation to the number of bacterial cells added at the beginning of digestion simulating the small intestine. This was caused by the necessity to ensure a lack of dependence on the different numbers of bacterial cells in the suspension formed from the material obtained at different time from the fecal microflora donors.

The digestion process of soy tempeh intensified the growth of Bifidobacterium and E. coli cells number, while bean tempeh rather stimulated the growth of E. coli and Enterococcus (Fig. 2). A higher increase in the counts of the tested bacteria was visible after digestion of cooked tempeh products than fried tempeh. Cooked beans and soy most stimulated the growth of number of bacterial cells from the genus Enteroccocus and E. coli and differences between stimulation of this group and Lactobacillus and Bifidobacterium was the largest in comparison with theses differences observed after digestion of other analyzed products. In digestion of the blank sample, where no product was added apart from water, a decrease was observed in the number of cells of the tested types of intestinal microflora, except for Enterococcus.

Applying the model simulating the transport of low molecular components to digest fried tempeh increased the stimulation of microflora growth (Fig. 3). Soy tem- 


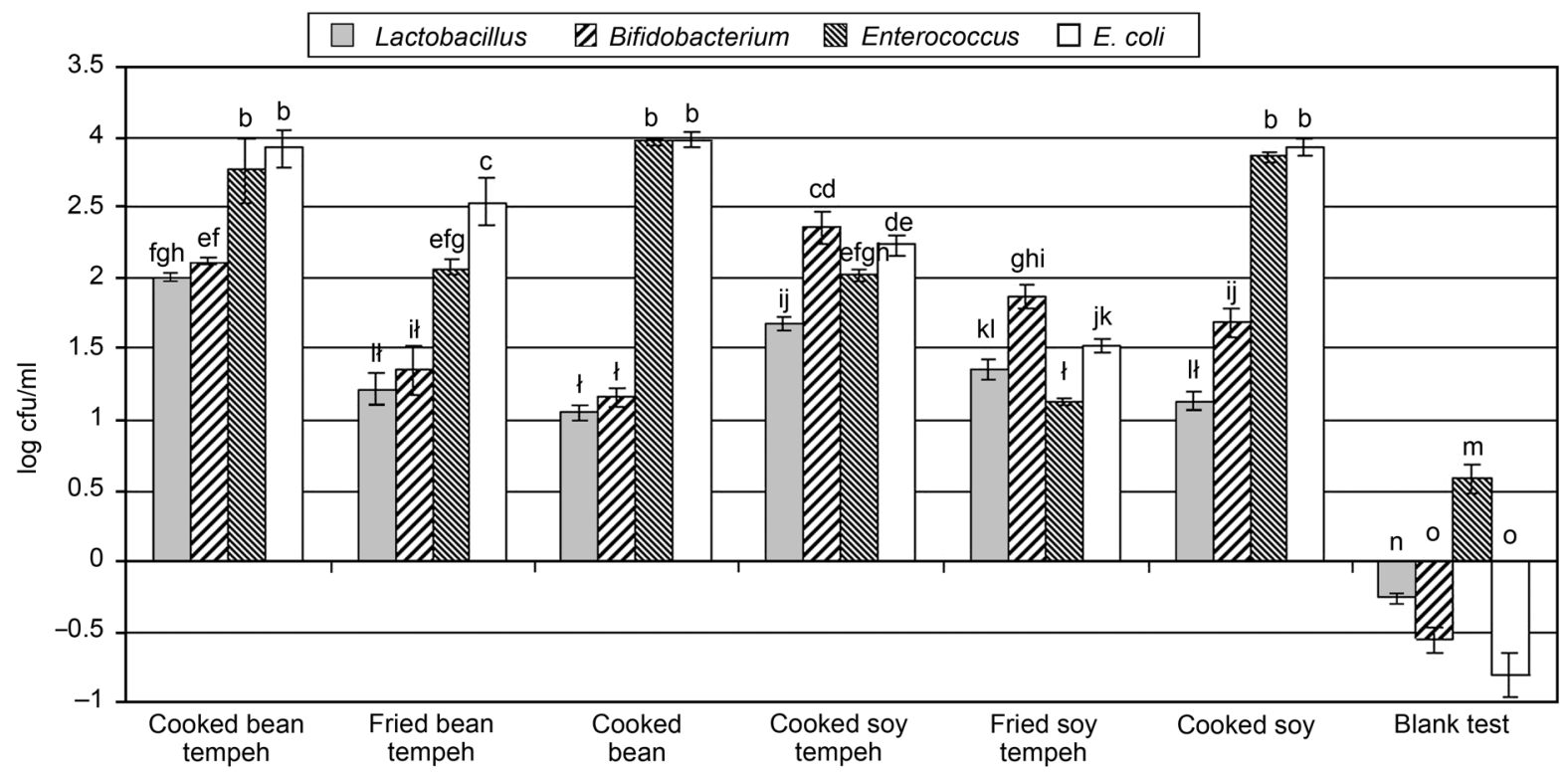

Fig. 2. Increments in the number of cells of intestinal microflora after digestion of tempeh products. Bars marked with different letters on the Fig. 2 and Fig. 3 differ significantly $(\mathrm{P}<0.05$; Tukey's test).

peh stimulated most the growth of bacteria from the genus Lactobacillus, and bean tempeh that of bacteria from the genera Bifidobacterium and E. coli. With the exception of the Lactobacillus bacteria, no statistically significant differences were found in growth stimulation of other marked types of intestinal microflora during digestion of both fried bean and soy tempeh. Applying the model simulating the transport of low molecular components to digest fried tempeh products, in comparison to the typical digestive system, led to increased growth of health-promoting intestinal microflora.

There have been many reports that after the consumption of soy tempeh the duration and incidence of diarrhea in humans (Karyadi and Lukito 2000) and animals (Karyadi and Lukito 1996; Karmini et al., 1997; Kiers et al., 2003) subsided. However, the mechanism behind this has not been clarified to date. It seems that it is not related to tempeh's antibacterial activity since in most cases thermal processing deactivated the antibacterial agent. Nowak and Steinkraus (1988) stated that subjecting pea tempeh to 10-minute cooking resulted in the complete disappearance of antibacterial properties against Clostridium perfringens. Roubos et al. (2008) observed the lack of antibacterial properties of a peptide isolated from tempeh after heating to $60^{\circ} \mathrm{C}$. Only Kobayasi et al. (1992) observed the antibacterial agent's resistance to temperature, but they tested a protein isolated from casein hydrolysate used in the R. oligosporus culture. The antibacterial agent isolated by Roubos et al. (2008) was susceptible to the action of proteases, thus digestive enzymes can also degrade the antibacterial peptides present in soy tempeh.

Other authors observed the effect of soy tempeh extracts on a decrease in adhesion of an enterotoxic

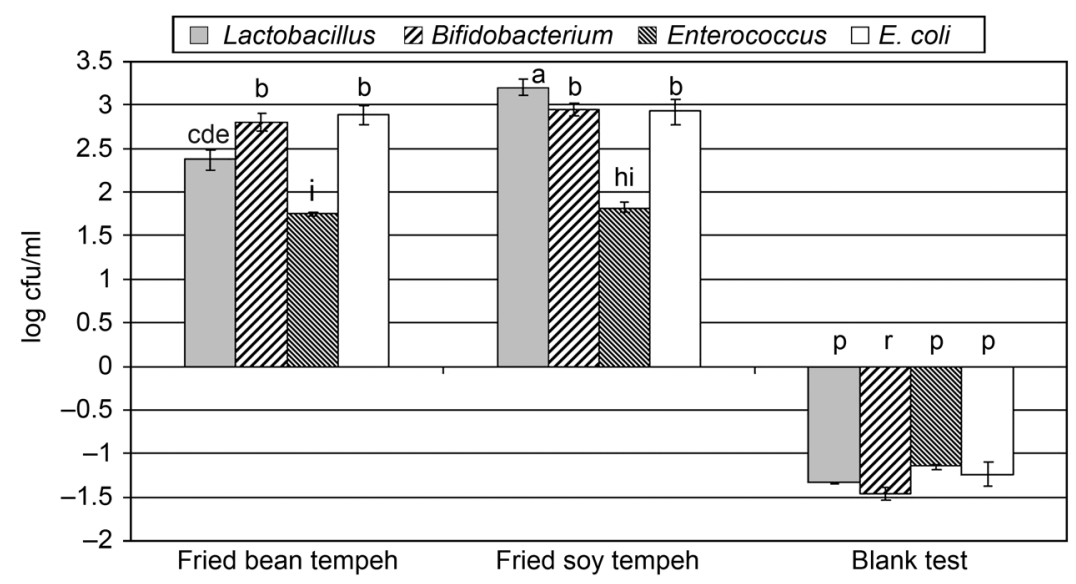

Fig. 3. Increments in the number of cells of intestinal microflora after digestion of tempeh products in the system with simulation of absorption of low melecular subtances.

Bars marked with different letters on the Fig. 2 and Fig. 3 differ significantly ( $\mathrm{P}<0.05$; Tukey's test). 
strain of E. coli to the intestinal epithelium cells of pigs (Kiers et al., 2002) and humans (Roubos et al., 2009), suggesting that this could be the antidiarrheal mechanism activated after tempeh consumption. However, tempeh used in the above-mentioned study was subjected neither to culinary processing nor to the digestion process, and these processes may to reduce these properties.

So far, an increase in intestinal bacteria counts has not been described during digestion of tempeh products. Stimulating growth of bacteria colonizing digestive tract microflora may comprise one of the mechanisms restoring the balance in the intestinal biotope and thus relieving the symptoms of diarrhea. Applying the in vitro digestion model stimulating absorption, which is more similar to conditions in the intestine, caused a growth of positive for human health microflora groups, however further studies on animals are necessary to confirm these results.

In conclusion, bean tempeh displayed antibacterial properties against $B$. subtilis, and no growth inhibition was observed in the remaining tested bacteria: E. coli, L. acidophillus and L. paracasei. When assessing intestinal bacteria counts in the in vitro digestion model, it was shown that tempeh products stimulated the growth of these bacteria, especially those from the genera Bifidobacterium and E. coli. Applying the model simulating the transport of low molecular components to in vitro digestion played a significant role in the increase of intestinal microflora counts. One of the mechanisms of tempeh products that prevents diarrhea might be the observed stimulation of growth of certain intestinal microflora groups.

\section{Acknowledgements}

This research was financially supported by the Polish Ministry of Science and Higher Education.

\section{Literature}

Aura A., S. Karpinen, H. Vritanen, P. Forssell, S. Heinonen, T. Nurmi, H. Adlercreutz and K. Poutanen. 2005. Processing of rye bran influences both the fermentation of dietary fibre and the bioconversion of lignans by human faecal flora in vitro. J. Sci. Food Agric. 85: 2085-2093.
Barry J., C. Hoebler, G. Macfarlane, S. Macfarlane, J. Mathers, K. Reed, P. Mortensen, I. Nordgaard, I. Rowlands and C. Rumney. 1995. Estimation of the fermentability of dietary fibre in vitro: a European interlaboratory study. Br. J. Nutr. 74: 303-322.

Goderska K., M. Gumienna and Z. Czarnecki. 2008. Release of phenolic compounds from bean flour, bean-derived chips and black chokeberry juice and changes in their activity during digestion in an in vitro gastrointestinal model. Pol. J. Food Nutr. Sci. 58: 497-501.

Gumienna M., M. Lasika and Z. Czarnecki. 2007. Effect of plant extract addition on phenolic compounds activity and intestinal microflora increase in the gastrointestinal tract model. Pol. J. Food Nutr. Sci. 57: 219-223.

Karmini M., E. Affandi, H. Hermana, D. Karyadi and F. Winarno. 1997. The inhibitory effect of tempe on Escherichia coli infection. Proceedings International Tempe Symposium. Indonesian Tempe Foundation. Bali. Indonesia, p. 157-162.

Karyadi D. and W. Lukito. 1996. Beneficial effects of tempeh in disease prevention and treatment. Nutr. Rev. 54: S94-S98.

Karyadi D. and W. Lukito. 2000. Functional food and contemporary nutrition-health paradigm: tempeh and its potential beneficial effects in disease prevention and treatment. Nutrit. 16: 697.

Kiers J., J. Meijer, M. Nout, F. Rombouts, M. Nabuurs and J. Meulen. 2003. Effect of fermented soy beans on diarrhea and feed efficiency in weaned piglets. J. Appl. Microbiol. 95: 545-552 .

Kiers J., M. Nout, F. Rombouts, M. Nabuurs and J. Meulen. 2002. Inhibition of adhesion of enterotoxigenic Escherichia coli K88 by soya bean tempe. Lett. Appl. Microbiol. 35: 311-315.

Kobayasi S., N. Okazaki and T. Koseki. 1992. Purification and characterization of an antibiotic substance produced from Rhizopus oligosporus IFO 8631. Biosci. Biotechnol. Biochem. 56: 94-98.

Marteau P., P. Pochart, J. Doré, C. Béra-Maillet, A. Bernalier and G. Corthier. 2001. Comparative study of bacterial groups within the human cecal and fecal microbiota. Appl. Environ. Microbiol. 67: 4939-4942.

Nout M. and J. Kiers. 2005. Tempe fermentation, innovation and functionality: update into the third millennium. J. Appl. Microbiol. 98: 789-805.

Nowak J. and K. Steinkraus. 1988. Effect of tempeh fermentation of peas on their potential flatulence productivity as measured by gas production and growth of Clostridium perfringens. Nutr. Rep. Int. 38: 1163-1172.

Roubos van den Hil P., E. Dalmas, R. Nout and T. Abee. 2008. Inactivation of Bacillus cereus cells and spores by low molecular weight proteinaceous compounds extracted from soybean tempeh. Abstracts of the 21st International ICFMH Symposium "Evolving microbial food quality and safety. FoodMicro 2008. Aberdeen. Scotland. p. 107.

Roubos van den Hil P., M. Nout, R. Beumer, J. Meulen and M. Zwietering. 2009. Fermented soya bean (tempe) extracts reduce adhesion of enterotoxigenic Escherichia coli to intestinal epithelial cells. J. Appl. Microbiol. 106: 1013-1021.

Wang H., D. Ruttle and C. Hesseltine. 1969. Antibacterial compound from a soybean product fermented by Rhizopus oligosporus. Proc. Soc. Exp. Biol. Med. 131: 579-583. 